\title{
Performance Analysis of Joint Base-Station Multiantenna Multibeam and Channel Assignment Scheme for Hierarchical Cellular System
}

\author{
Peng Yang, ${ }^{1,2}$ Dou Liu, ${ }^{2}$ and Yan Zhang' \\ ${ }^{1}$ China Academy of Telecommunications Research, MIIT, Beijing 100191, China \\ ${ }^{2}$ Chongqing University of Posts and Telecommunications, Chongqing 400065, China \\ Correspondence should be addressed to Peng Yang; yangpeng@catr.cn
}

Received 19 February 2014; Accepted 18 June 2014; Published 7 July 2014

Academic Editor: Xiang Zhang

Copyright (c) 2014 Peng Yang et al. This is an open access article distributed under the Creative Commons Attribution License, which permits unrestricted use, distribution, and reproduction in any medium, provided the original work is properly cited.

Different from the traditional configuration of hierarchical cellular, we introduce the joint base-station multi-antenna multi-beam and channel assignment scheme for hierarchical cellular in this paper. The proposed scheme is based on multi-beam base-station antenna splitting in the elevation-radiating plane, and a dynamic channel allocation scheme by combining the adaptive antenna technologies. Simulation results show that the proposed configuration of the hierarchical cellular can enhance the spectral efficiency remarkably.

\section{Introduction}

Recently, considerable research efforts have been put into hierarchical cellular system and dynamic channel assignment [1-7]. In order to alleviate the mobile fundamental contradiction between the limited frequency resources of mobile communication and the growing number of users, the importance and necessity of research on the application of radio resource management are becoming more apparent. The effective use of the adaptive antennas and channel resources can resolve the abovementioned contradiction [8-14]. In the past decade, the adaptive antennas have been proposed to improve the spectrum efficiency and provide a new approach to the next-generation networks, which have been considered as one of most important technologies in the wireless communications [15-18]. By adopting the basestation adaptive antenna technology [19-21], intelligent cell with adaptive array antennas was proposed [22-24]. On the one hand, the beam can be split or the multibeam method can be adopted to reduce the influence caused by the radio propagation environment, which leads to the improved channel quality and the enhanced system capacity. On the other hand, flexible intercell handover can be realized by controlling antenna beam coverage and transmission power intelligently $[25,26]$. The base-station adaptive antennas are adopted to monitor the location of the mobile station and the antenna beam is adopted to track the mobile station. Thus the path can be determined, on which the mobile station can be connected under the limited power. In general, this is an effective method to enhance the system capacity by combining the adaptive antenna technology with the cell technology. An intelligent cell with switch beam is introduced in $[17,27,28]$. In this system, all the beams of the basestation adaptive antennas share available channels and the antenna system is responsible for determining which beam the current location of the mobile station belongs to; thus it uses the antenna switch to connect the radio channel to the beam, correspondingly. Formally, this intelligent cell makes sectorization within cell and the mobile station does not need to handover among beams. Thus, this kind of cell can not only reduce the times of handover but also enhance the route efficiency and system capacity. However, the intelligent needs to track the mobile station, so it is inevitable to be influenced by the number and size of antenna elements, and the capability of reducing co-channel interference and the flexible handover capability are limited. 

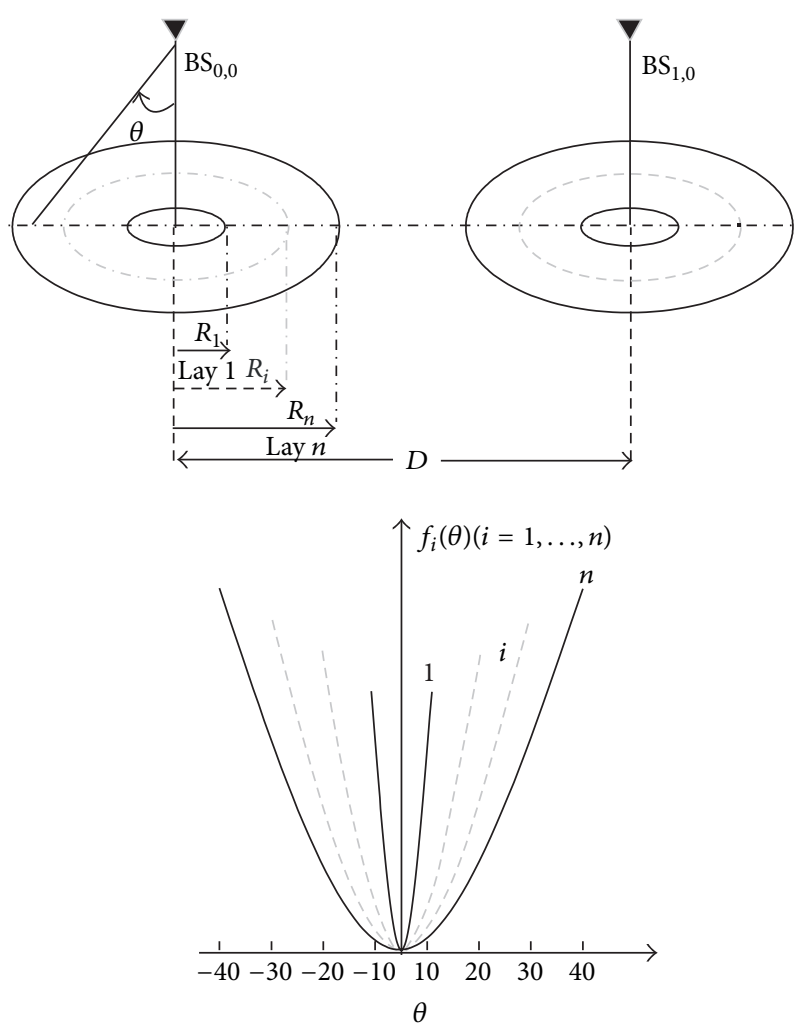

FIgURE 1: Adaptive multibeam antenna hierarchical cellular structure.

Many hierarchical cellular mechanisms have been proposed for various communication systems with high channel capacity [29-33]. In this paper, we focus on the hierarchical cellular joint base-station multiantenna multibeam and channel assignment. By combining the adaptive antenna technology, mobile communication cellular technology, dynamic channel allocation fusion concept, and the coverage uniformity description, a vertical adaptive multiantenna technology and a dynamic channel allocation scheme are exploited to design the hierarchical cellular.

\section{Cell Structure Model}

We use the adaptive multi-beam antenna in the base station to form the cluster of concentric circles, whose center is the base station by using the vertical division and antenna pitch plane which is shown in Figure 1. Each cell consists of $n$ concentric whose radii are $\left[0, R_{1}\right],\left[0, R_{2}\right], \ldots,\left[0, R_{n}\right]$, and each concentric circle is regarded as a hierarchical cellular. We divided this $n$ concentric to $n$ layers according to the radius of each concentric. For example, the smallest one is the first layer, followed by the second layer,..., and the largest one is the $n$th layer, and each of them is marked by Lay 1 , Lay $2, \ldots$, Lay $n$, and the $i$ th layer is formed by the $i$ th beam of the base station with multibeam antenna as shown in Figure 1.

In fact, in order to make sure that each layer of the cell has uniform coverage or that the mobile station has the same received power, we normalize the horizontal beam pattern.
Thus, we can use the elevation beam pattern to gain the $i$ th beam pattern $f_{i}(\theta)[34]$ :

$$
f_{i}(\theta)=\left(\frac{h_{b}}{\cos \theta}\right)^{\alpha}
$$

where $\alpha$ is the propagation loss factor, $h_{b}$ is the height of basestation antenna, and $\theta=\tan ^{-1}\left(R_{i} / h_{b}\right)$.

\section{Channel Allocation Scheme}

In Figure 2, a hierarchical cellular system with $n$ layers is established in terms of the method shown in Figure 1. As we can see in Figure 2, the number of cell clusters or the frequency reuse factor is $N$ (a cell group consists of $N$ cells.). We suppose that the reference cell group is shown in the Figure 2 that the layers from inner to outer are the layer 1 interference cell group, layer 2 interference cell group and so on.

Figure 2 describes the frequency allocation assignment. We stipulate that the channel group $C_{k, j}$ of the reference cell $\mathrm{BS}_{0,0}$ is the channel group whose region is annular region $\left[R_{i-1}, R_{i}\right],(k=1, \ldots, N i=1, \ldots, N)$. The adjacent frequency region group use the same frequency but the channel group allocation is opposite. In the same cell, the Layj can use the channel of Lay $i$, but Lay $i$ cannot use the channel of Lay $j$, where $i>j$. As shown in Figure 2, channel allocation in each layer of the reference cell $\mathrm{BS}_{0,0}$ from the first layer to the $n$th layer is $\left\{C_{1,1}, C_{1,1}+C_{1,2}, \ldots, C_{1,1}+\cdots+C_{1, n}\right\}$. Thus in the same frequency adjacent cell (such as $\mathrm{BS}_{1,0}$ ), the channel allocation in each layer from the first one to the $n$th one is $\left\{C_{1, n}, C_{1, n}+C_{1, n-1}, \ldots, C_{1, n}+\cdots+C_{1,1}\right\}$.

In order to make the distance of the adjacent areas within the same frequency group be the longest, the priority of channel is provided as follows.

(1) Within the same cell, Lay $j$ should first use the $j$ th group of channel; namely, the call users of Lay $j$ should use the group $C_{k, j}$.

(2) Lay $j$ can use the channel of Lay $i(i<j)$ only when the channels of $C_{k, j}$ are all used and there are idle channels in $C_{k, i}$.

(3) When Lay $j$ and Lay $i$ choose the channels of $C_{k, i}$, where $i<j$. Lay $i$ has the priority.

(4) We assume that each layer of two adjacent regions with the same frequency uses the same channel whose beam covering forms concentric circles with radii $R_{i}$, $R_{j}$, and $i+j=n+1(i, j=1, \ldots, n)$, where denotes the order item of layers from inner to outer in the same cell.

\section{Choice of Radius and Channel for Each Layer}

In order to know the relationship between the throughput and channel occupancy in cell, we first make the following definitions. We assume that the throughput density per unit area is $\lambda$, and then the probability that there are $m$ users in 


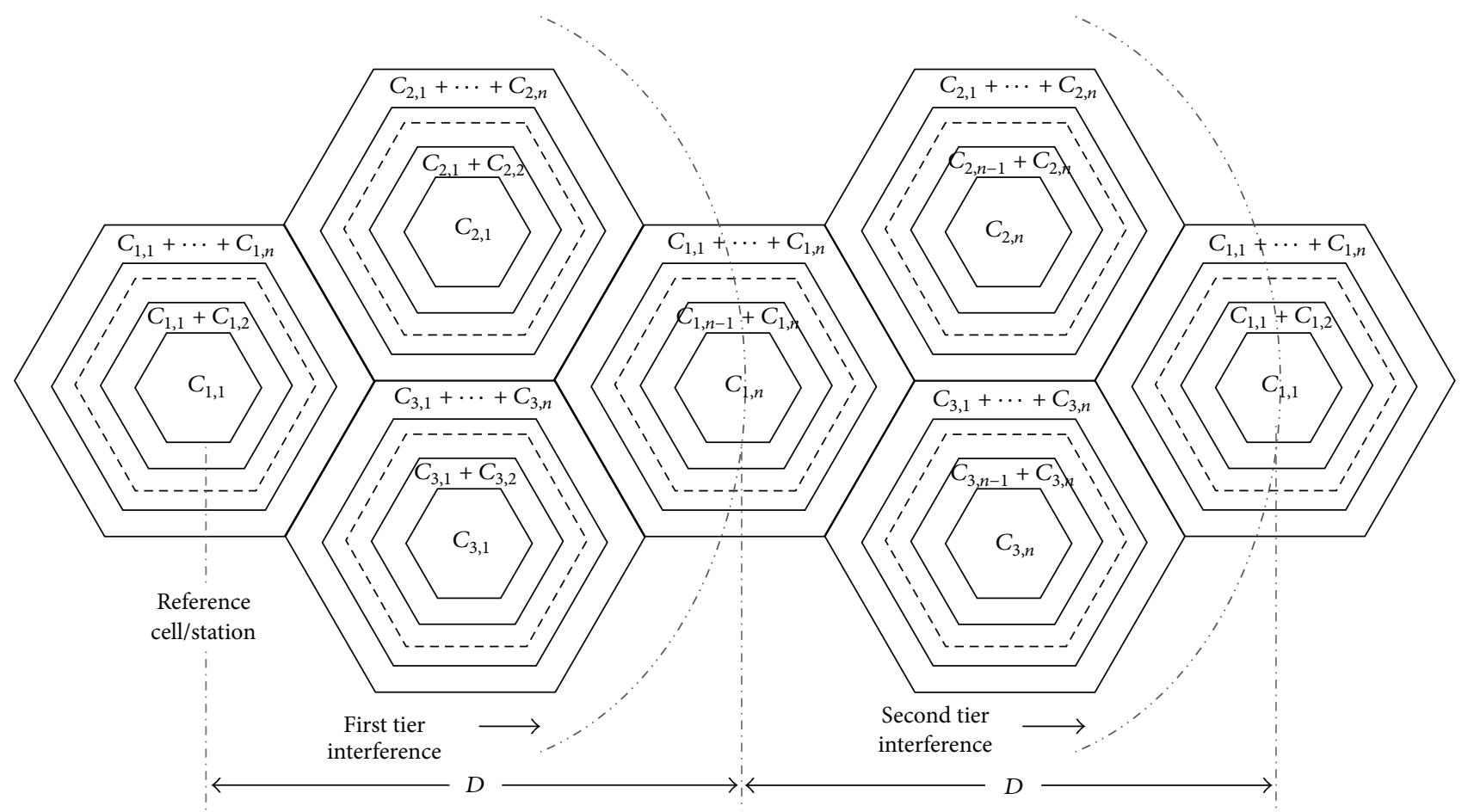

FIGURE 2: The structure of the adaptive multibeam antenna hierarchical cellular group.

the region of the disc is $P(m, A)$ which satisfies the Poisson distribution [35-37]:

$$
P(m, A)=\frac{(\lambda A)^{m}}{m !} e^{-\lambda A}
$$

The area of the circle whose radius is $R$ should be $A=$ $\pi R^{2}$. Thus we assume that $A_{i}=\pi R_{i}^{2}$ should be the area of the circle whose radius is $R_{i}$ (where $R_{i}<R$ ). So the probability that there are $m$ users in the area whose radius is $R_{i}$ :

$$
\begin{aligned}
P\left(\mathbb{R}_{i} \leq R_{i}\right) & =P\left\{A_{i} \leq A \mid A \leq \pi R^{2}\right\} \\
& =\frac{P\left\{A_{i} \leq A, A \leq \pi R^{2}\right\}}{P\left\{A \leq \pi R^{2}\right\}} .
\end{aligned}
$$

Consider $R_{i} \leq R$ :

$$
\begin{aligned}
& P\left\{A_{i} \leq \pi R_{i}^{2}, A \leq \pi R^{2}\right\}=P\left\{A \leq \pi R_{i}^{2}\right\} \\
f_{\mathbb{A}}(A)= & \frac{d(1-P(m, A))}{d A}=\frac{d\left(1-\sum_{l=0}^{m}\left((\lambda A)^{l} / l !\right) e^{-\lambda A}\right)}{d A} \\
= & \sum_{l=0}^{m}\left(\lambda \frac{(\lambda A)^{l-1}}{(l-1) !} e^{-\lambda A}-\lambda \frac{(\lambda A)^{l}}{l !} e^{-\lambda A}\right) \\
= & \lambda \frac{(\lambda A)^{m-1}}{(k-1) !} e^{-\lambda A} .
\end{aligned}
$$

Then

$$
\begin{aligned}
P\left\{\mathbb{R}_{i} \leq R_{i}\right\} & =\frac{P\left\{A_{i} \leq \pi R_{i}^{2}\right\}}{P\left\{A \leq \pi R^{2}\right\}} \\
& =\frac{\int_{0}^{\pi R_{i}^{2}} \lambda\left((\lambda \alpha)^{m-1} /(k-1) !\right) e^{-\lambda \alpha} d \alpha}{\int_{0}^{\pi R^{2}} \lambda\left((\lambda \alpha)^{m-1} /(k-1) !\right) e^{-\lambda \alpha} d \alpha} \\
& =\frac{1-\sum_{l=0}^{m}\left(\left(\lambda \pi R_{\mathrm{i}}^{2}\right)^{l} / l !\right) e^{-\lambda \pi R_{i}^{2}}}{1-\sum_{l=0}^{m}\left(\left(\lambda \pi R^{2}\right)^{l} / l !\right) e^{-\lambda \pi R^{2}}} .
\end{aligned}
$$

Thus

$$
\begin{aligned}
f_{\mathbb{R}_{i}}\left(R_{i}\right)= & \frac{d\left[P\left\{\mathbb{R}_{i} \leq R_{i}\right\}\right]}{d R_{i}} \\
= & \left(\sum_{l=0}^{m} e^{-\lambda \pi R_{i}^{2}}\left(d\left(\lambda \pi R_{i}^{2}\right) / d R_{i}\right)\right. \\
& \times\left[l \cdot\left(\left(\lambda \pi R_{i}^{2}\right)^{i-1} /(m-1) !\right)\right. \\
& \times\left(1-\sum_{l=0}^{m}\left(\left(\lambda \pi R^{2}\right)^{l} / l !\right) e^{-\lambda \pi R^{2}}\right)^{-1} \\
= & \frac{\lambda \pi R_{i}^{2} \cdot\left(\left(\lambda \pi R_{i}^{2}\right)^{m-1} /(k-1) !\right) e^{-\lambda \pi R_{i}^{2}}}{1-\sum_{l=0}^{m}\left(\left(\lambda \pi R^{2}\right)^{l} / l !\right) e^{-\lambda \pi R^{2}}} .
\end{aligned}
$$




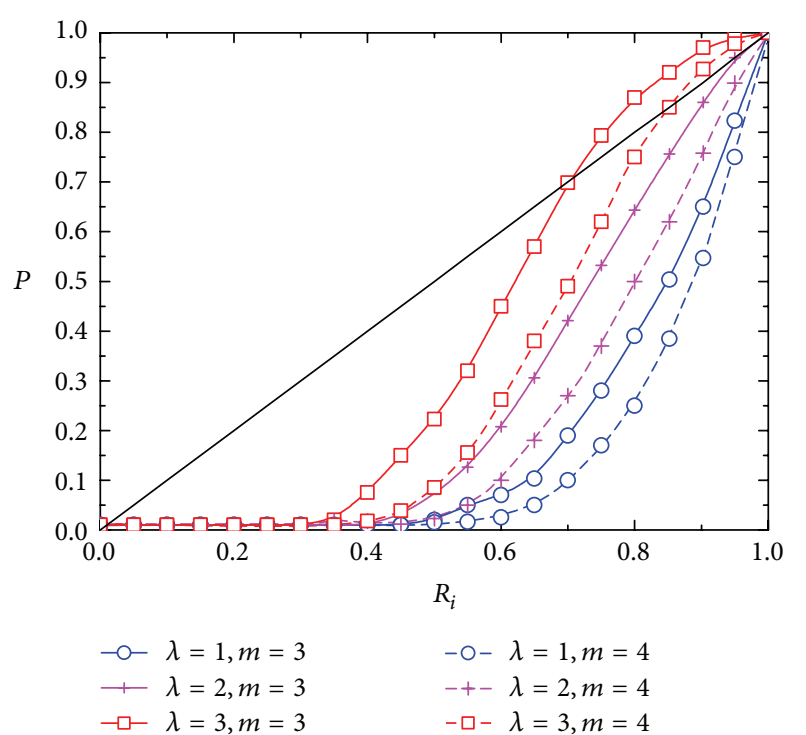

FIgURE 3: Relationship between the hierarchical radius and the number of channels and the throughput density.

The simulation results about the relationship between a group of hierarchical radius and the throughput density are shown in Figure 3. The horizontal axis is $R_{i}$ 's normalized position in $R$, and the vertical axis is the different probabilities with different numbers of channels $m$ and throughput densities $\lambda$. It can be seen in Figure 3 that in order to reach the same probability with the same amount of channels, the radius is becoming shorter from inner to outer with the increase of throughput density. Thus we can infer that the distribution of the users is gradually becoming nonuniform, and the layers are becoming closer from outer to inner. Furthermore, the radius of layer is becoming longer when the number of the channels is becoming larger. In other words, when there are more channels, there are more users, so in order to make sure that there are the same number of users in the same unit areas, it is necessary to increase the radius, namely, the area.

In order to implement the channel allocation scheme described above, we use an approximate method to determine the initial set of channel and the channel allocation within the group. Assume that the disc whose radius is $R$ is divided into $n$ layers, and there are $m$ channels in each layer. In other words, the total number of channels in disc is $m n$. The first layer's radius is $R_{1}$ and the probability that $n m$ channels are allocated in this layer is $1 / n$. And the second layer's radius is $R_{2}$ and the probability that there are $m n$ channels in this layer is $2 / n$. And the $i$ th layer's radius is $R_{i}$ and the corresponding probability that there are $m n$ channels in this layer is $i / n$ and so on. Obviously, if we adopt this method to divide the initial hierarchical radius, we can meet the requirements that are required in the channel allocation and the hierarchical radius. Thus, we can use the following steps to determine the hierarchical radius.

Step 1. Check the total number of channels and the throughput density in cell.
Step 2. Calculate the probability by using formula (2).

Step 3. Determine the number of layers $n$, and determine $R_{i}$ which is used in calculating the probability $P\left\{R_{i} \leq i / n\right\}$; then the radius of $i$ th layer $R_{i}$ will be confirmed.

Step 4. Return to Step 1 when the number of channels or the number of users is changed.

Figure 4 shows a set of computer simulation results to determine the hierarchical radius. When the number of layer is 5 , the approximate radius would change with the number of initial set of channels or the different throughput densities. It can be seen from Figure 4 that in order to reach the same probability, the hierarchical radius from outer to inner are becoming smaller with the increase of throughput density under the condition of the same amount of channels. At the same time, the hierarchical radius increases with the growth of channels with the same throughput density. In conclusion, the simulation results from Figure 3 and Figure 4 are consistent, which proves that our approach is significant.

\section{The Impact of the Same Frequency Interference on the Bandwidth Efficiency}

Based on the structure of cell and the channel allocation scheme described above, we set a cochannel frequency interference analysis model as shown in Figure 5. To make the analysis easy, we assume that the average distance between the location of the users in the $i$ th layer of $\mathrm{BS}_{0,0}$ and the center of $\mathrm{BS}_{0,0}$ is $\widetilde{R}_{0, i}$. Meanwhile, we assume that the average distance between the center of one of the cochannel frequency interference base stations $\mathrm{BS}_{1,0}$ in the first layer of interference sources and the users in the ith layer of the $\mathrm{BS}_{0,0}$ is $\widetilde{R}_{1, i}$, and

$$
\widetilde{R}_{0, i} \simeq \int_{R_{i-1}}^{R_{i}} P(x, n m) x d x,
$$

where $P(x, n m)$ denotes the probability that there are $n m$ channels in the layer whose radius is $x$ :

$$
\widetilde{R}_{1, i} \simeq D-\widetilde{R}_{0, i}
$$

Thus, we can use the following formula to denote the useful signal power received by the users in the $i$ th layer of $\mathrm{BS}_{0,0}$ :

$$
P_{0, i}=P_{T} \widetilde{r}_{0, i}^{-\alpha},
$$

where $P_{T}$ is the transmission power of $\mathrm{BS}_{0}$, and it is normalized to be 1 .

Here, Figure 5 takes $\mathrm{BS}_{0,0}$ and $\mathrm{BS}_{1,0}$ as examples to analyze the interference of the same frequency. As previously explained in Section 4, the cochannel frequency interference impact on the users in the $i$ th layer of $\mathrm{BS}_{0,0}$ is from the layer of $\mathrm{BS}_{1,0}$ whose label is $(n+1-i)$. And according to the channel allocation scheme described above, we can know that the channels in the $i$ th layer of $\mathrm{BS}_{0,0}$ are borrowed from the $j$ th 

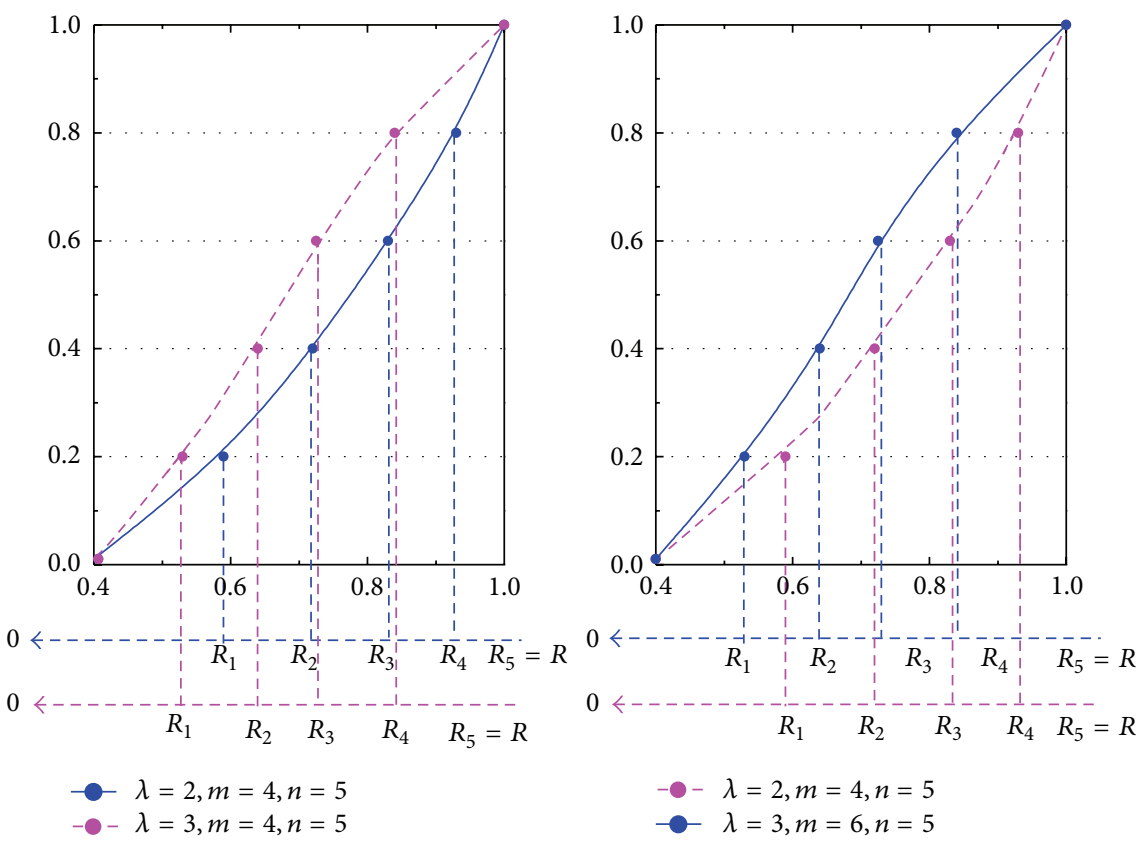

FIGURE 4: The relationship among the division of the hierarchical radius and the number of channels and throughput density.

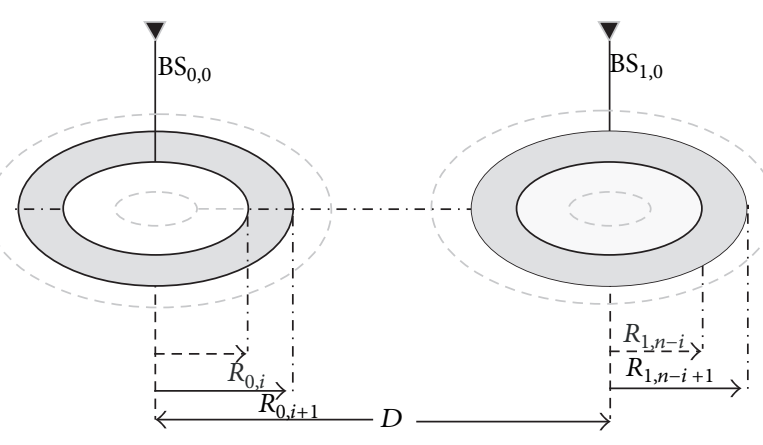

FIGURE 5: Cochannel frequency interference analysis model.

layer $(j=1, \ldots, i-1)$, and the channels in the $j$ th layer are lent to $(n+1-i)$ th $(j=1 \ldots, n-i)$ layer of $\mathrm{BS}_{1,0}$. Therefore, whether the $i$ th layer of $\mathrm{BS}_{0,0}$ borrows channels or not, and whether the $(n+1-i)$ th layer of $\mathrm{BS}_{1,0}$ lends channels or not will determine the channel allocation results. Next, the analysis of these four different cases are as following.

(1) $\mathrm{BS}_{0,0}$ borrows channels and $\mathrm{BS}_{1,0}$ does not lend the channels.

If the $i$ th layer of $\mathrm{BS}_{0,0}$ borrows the channels from the $j$ th layer, it must meet the conditions that there are $m+1$ users at the same time, and all the $m$ channels in the $l$ th $(l=j+$ $1, \ldots, i-1)$ layer are occupied and there is at least one of $m$ channels in the $j$ th layer that is not occupied. What is more, if the layers in $\mathrm{BS}_{1,0}$ do not lend the channels, it must meet the condition that it is not necessary for the layers from $(n+2-i)$ to $n$ to borrow channels:

$$
\begin{aligned}
& I_{y n}=\underbrace{P(n-i+2, m) * P(n-i+3, m) * \cdots * P(n-1, m) P(n, m)}_{\text {the channels in the }(n-i+1) \text { th layer of } \mathrm{BS}_{1,0} \text { are not borrowed }}
\end{aligned}
$$

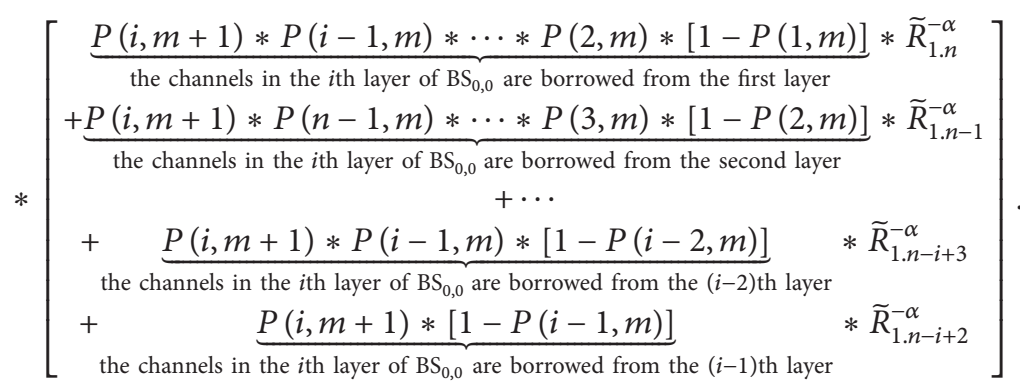


(2) $\mathrm{BS}_{0,0}$ does not borrow the channels and $\mathrm{BS}_{1,0}$ lends the channels:

$$
\begin{aligned}
& I_{n y}=\underbrace{P(1, m) * P(2, m) * \cdots * P(i-2, m) P(i-1, m)}_{\text {the } i \text { th layer of } \mathrm{BS}_{0,0} \text { does not borrow channels }}
\end{aligned}
$$

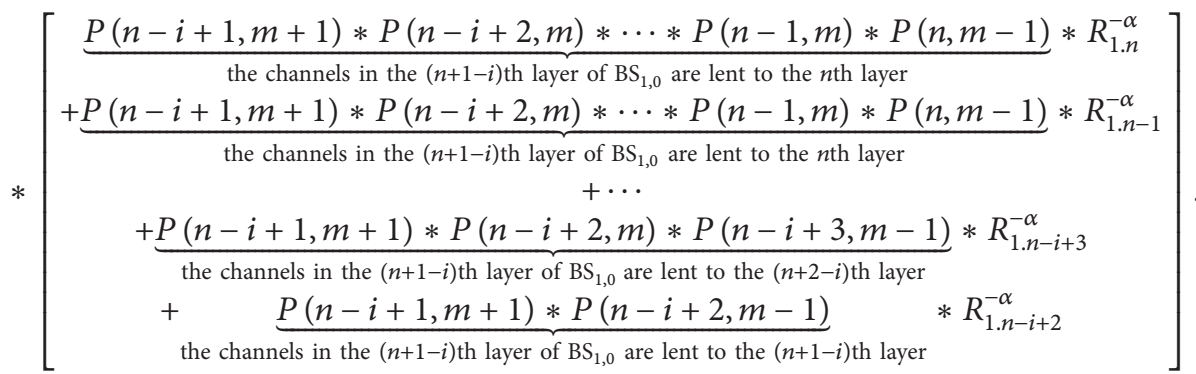

(3) $\mathrm{BS}_{0,0}$ does not borrow the channels and $\mathrm{BS}_{1,0}$ does not lend the channels:

$$
\begin{aligned}
I_{n n}= & \underbrace{P(n-i+2, m) * P(n-i+3, m) * \cdots(n, m)}_{\text {the channels in the }(n-i+1) \text { th layer of } \mathrm{BS}_{1,0} \text { are not lent }} \\
& * \underbrace{P(1, m) * P(2, m) * \cdots * P(i-2, m) * P(i-1, m)}_{\text {the } i \text { th layer of } \mathrm{BS}_{0,0} \text { does not borrow channels }} * \widetilde{R}_{1, i}^{-\alpha} .
\end{aligned}
$$

(4) $\mathrm{BS}_{0,0}$ borrows the channels and $\mathrm{BS}_{1,0}$ does not lend the channels

$$
\begin{aligned}
& I_{y y}=\underbrace{[1-P(n-i+2, m) * P(n-i+3, m) * \cdots(n, m)]}_{\text {the channels in the }(n-i+1) \text { th layer of } \mathrm{BS}_{1,0} \text { are lent }}
\end{aligned}
$$

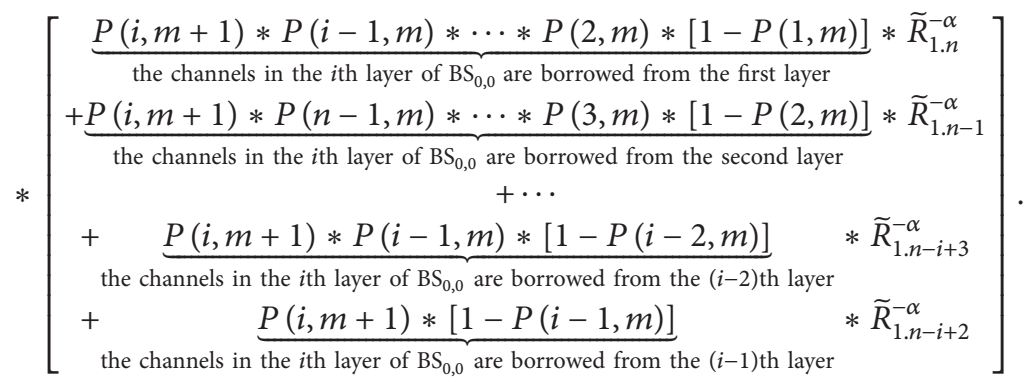

Assuming that the interference sources have the equal influence and only the cochannel frequency interference sources in the first layer of $\mathrm{BS}_{0,0}$ are considered, If the frequency reuse factor is $N$, then the signal to noise ratio $\mathrm{CIR}_{0, i}$ in the $i$ th layer of $\mathrm{BS}_{0,0}$ can approximately be

$$
\mathrm{CIR}_{0, i}=\frac{\tilde{r}_{0, i}^{-\alpha}}{(N-1)\left[I_{y y}+I_{y n}+I_{n y}+I_{n n}\right]} .
$$

The channel capacity $\mathbb{C}_{0, i}$ in the $i$ th layer of $\mathrm{BS}_{0,0}$ follows the Shannon theorem as given in

$$
\mathbb{C}_{0, i}=W_{0, i} \log \left(1+\mathrm{CIR}_{0, i}\right),
$$

where $W_{0, i}$ is bandwidth provided for the $i$ th layer of $\mathrm{BS}_{0,0}$.

Thus, the channel capacity $\mathbb{C}_{0}$ of $\mathrm{BS}_{0,0}$ is

$$
\mathbb{C}_{0}=\sum_{i=1}^{n} W_{0, i} \log \left(1+\mathrm{CIR}_{0, k}\right) \text {. }
$$




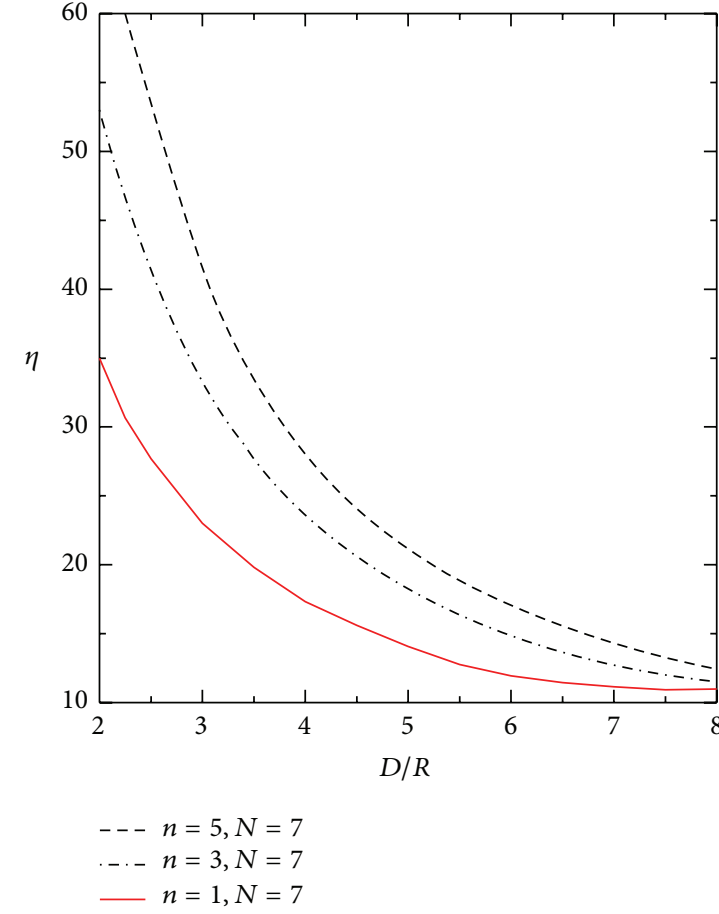

(a)

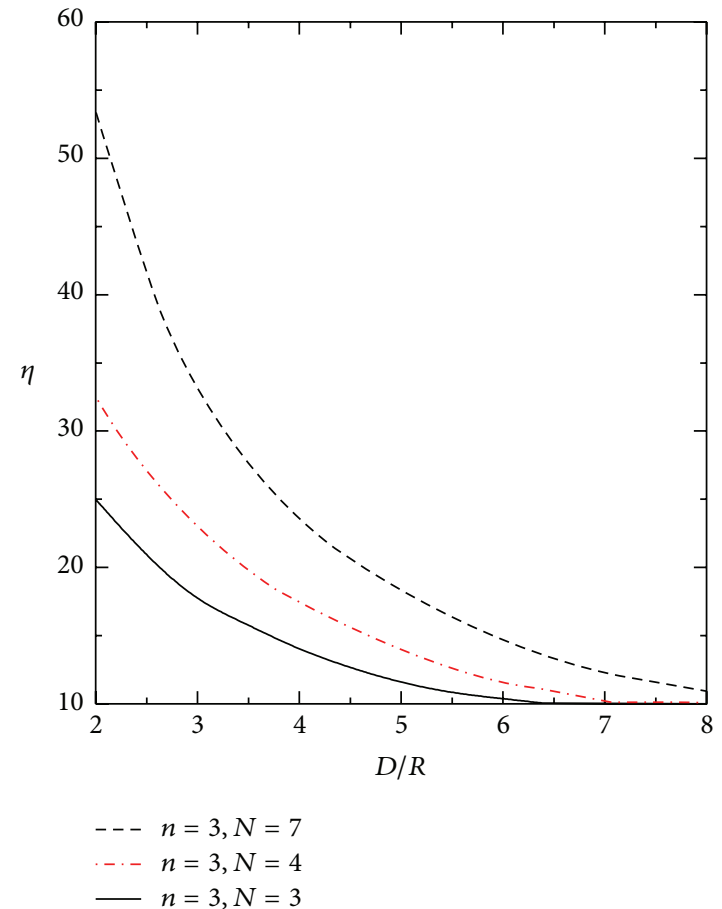

(b)

FIGURE 6: The relationship between $D / R$ and $\eta$.

Therefore, the spectral efficiency $\eta$ of $\mathrm{BS}_{0,0}$ can be calculated as

$$
\eta=\frac{\mathbb{C}_{0}}{\pi W_{0} R^{2}}
$$

where $W_{0}$ is the bandwidth provided for $\mathrm{BS}_{0,0}$.

Figure 6 shows the relationship between the spectral efficiency $\eta$ and the cochannel frequency reuse ratio $D / R$. The parameter selection is as follows: the propagation loss factor is $a=4$, and the height of the base-station antenna is $h_{b}=10 \mathrm{~m}$, $R=200 \mathrm{~m}$, and throughput density is 1 . It can be seen from Figure $6(\mathrm{a})$ that $n=1$ indicates that the cell is not divided, and the spectral efficiency $\eta$ is enhanced by layered cell and channel allocation. And it can be seen from Figure 6(b) that when the number of layers is the same, the spectral efficiency $\eta$ is improved with the increase of $D / R$.

\section{Conclusion}

In this paper, the hierarchical cellular joint base-station multiantenna multibeam and channel assignment scheme are introduced. Simulation results can prove that this kind of structure of the cell can be an effective method to enhance the spectral efficiency and solve the dynamic hierarchical problems without too much considering the cochannel interference that resulted from it. The key point of the future work is to combine the latest researching progress of multiantenna such as MIMO. Meanwhile, we will study how to take better advantages of the technologies which are applied in the cells to enhance the efficiency of coverage and hence enhance the efficiency and credibility of the transmission system. In the future, we will try our best to find ways to shorten the width of beams and improve the flexibility of beams. All of these are prepared to provide more effective technical support for making the intelligent cell technology come true.

\section{Conflict of Interests}

The authors declare that there is no conflict of interests regarding the publication of this paper.

\section{Acknowledgment}

The authors would like to acknowledge the National Science and Technology Major Project of the Ministry of Science and Technology of China (2012ZX03001014, 2013ZX03001007, and 2014ZX03001020-003).

\section{References}

[1] N. K. Mishra, D. K. Vishwakarma, and A. Kumar, "Performance analysis of micro-strip patch antenna and dielectric resonator antenna array," in Proceedings of the International Conference on Emerging Trends in Computing, Communication and Nanotechnology (ICE-CCN '13), pp. 721-726, IEEE, March 2013.

[2] G. Okamoto, S. S. Jeng, S. Tseng, and G. Xu, "Evaluation of beamforming algorithm effectiveness for the smart wireless lan system," in Proceedings of the 48th IEEE Vehicular Technology Conference (VTC'98), vol. 3, 1998. 
[3] H. Shajaiah, A. Khawar, A. Abdel-Hadi, and T. C. Clancy, "Resource allocation with carrier aggregation in LTE Advanced cellular system sharing spectrum with S-band radar," in Proceedings of the IEEE International Symposium on Dynamic Spectrum Access Networks (DYSPAN '14), 2014.

[4] E. Axell, G. Leus, E. G. Larsson, and H. V. Poor, "Spectrum sensing for cognitive radio: state-of-the-art and recent advances," IEEE Signal Processing Magazine, vol. 29, no. 3, pp. 101-116, 2012.

[5] L. Fangwei and L. Guobiao, "Capacity performance analysis of TD-HSUPA system based on a new hierarchical cellular," in Proceedings of the 4th International Conference on Intelligent Computation Technology and Automation (ICICTA '11), vol. 2, pp. 1070-1073, Shenzhen, China, March 2011.

[6] C. W. Sung and W. S. Wong, "User speed estimation and dynamic channel allocation in hierarchical cellular system," in Proceedings of the 44th IEEE Vehicular Technology Conference, vol. 1, pp. 91-95, Stockholm, Sweden, June 1994.

[7] B. Jabbari and W. F. Fuhrmann, "Teletraffic modeling and analysis of flexible hierarchical cellular networks with speedsensitive handoff strategy," IEEE Journal on Selected Areas in Communications, vol. 15, no. 8, pp. 1539-1548, 1997.

[8] S. Jeng, G. T. Okamoto, G. Xu, H. Lin, and W. J. Vogel, "Expérimental evaluation of smart antenna system performance for wireless communications," IEEE Transactions on Antennas and Propagation, vol. 46, no. 6, pp. 749-757, 1998.

[9] R. Matsukawa, T. Obara, and F. Adachi, "A dynamic channel assignment scheme for distributed antenna networks," in Proceedings of the 75th Vehicular Technology Conference, (VTC '12), IEEE, Yokohama, Japan, May 2012.

[10] A. Khanafer, T. J. Lim, R. Doostnejad, and T. Tang, "MIMOOFDMA rate allocation and beamformer design using a multiaccess channel framework," in Proceedings of the IEEE International Conference on Communications (ICC '12), pp. 2553-2558, Ottawa, Canada, June 2012.

[11] L. Song, M. Peng, and Y. Li, "Resource allocation for vertical sectorization in LTE-advanced systems," International Journal of Antennas and Propagation, vol. 2013, Article ID 456760, 7 pages, 2013.

[12] R. Matsukawa, T. Obara, and F. Adachi, "A dynamic channel assignment scheme for distributed antenna networks," in Proceedings of the 75th IEEE Vehicular Technology Conference (VTC '12), pp. 1-5, 2012.

[13] A. Khanafer, T. J. Lim, R. Doostnejad, and T. Tang, "MIMOOFDMA rate allocation and beamformer design using a multiaccess channel framework," in Proceedings of the IEEE International Conference on Communications (ICC '12), pp. 2553-2558, June 2012.

[14] S. L. Chen, P. H. J. Chong, and M. Yang, "Dynamic channel assignment with flexible reuse partitioning in cellular systems," Wireless Personal Communications, vol. 42, no. 2, pp. 161-183, 2007.

[15] L. C. Godara, "Application of antenna arrays to mobile communications, part II: beam-forming and direction-of-arrival considerations," Proceedings of the IEEE, vol. 85, no. 8, pp. 11951245, 1997.

[16] A. R. Lopez, "Performance predictions for cellular switchedbeam intelligent antenna systems," IEEE Communications Magazine, vol. 34, no. 10, pp. 152-154, 1996.

[17] M. Ho, G. L. Stiiber, and M. D. Austin, "Performance of switched-beam smart antennas for cellular radio systems," IEEE Transactions on Vehicular Technology, vol. 47, no. 1, pp. 10-19, 1998.
[18] A. Lozano, A. Goldsmith, R. A. Valenzuela, M. A. Lagunas, and D. Gesbert, "Guest editoria-Advances in smart antennas," IEEE Wireless Communications, vol. 13, no. 4, pp. 6-7, 2006.

[19] A. Kanazawa, H. Harada, T. Iwama, and Y. Hase, "The feasibility study of the dynamic zone configuration technique with a developed circular array antenna," IEICE Transactions on Fundamentals of Electronics, Communications and Computer Sciences, vol. 82, no. 7, pp. 1210-1221, 1999.

[20] J. Zhang, B. Li, and J. Liu, "An efficient adaptive cell sectoring technique for non-uniform traffic in DS-CDMA systems," Wireless Networks, vol. 12, no. 3, pp. 345-355, 2006.

[21] W. Guo, J. M. Rigelsford, K. L. Ford, and T. O’Farrell, "Dynamic basestation antenna design for low energy networks," Progress in Electromagnetics Research C, vol. 31, pp. 153-168, 2012.

[22] M. Kitahara, Y. Ogawa, and T. Ohgane, "A base station adaptive antenna for downlink transmission in a DS-CDMA system," in Proceedings of the IEEE 51st Vehicular Technology Conference (VTC'00), vol. 2, pp. 710-715, Tokyo, Japan, 2000.

[23] K.-K. Wong and R.-S. Cheng, "Adaptive antennas at the mobile and base stations in an OFDM/TDMA system," IEEE Transactions on Communications, vol. 49, no. 1, pp. 195-206, 2001.

[24] Y. Wang and J. R. Cruz, "Performance enhancement of CDMA cellular systems with augmented antenna arrays," IEEE Journal on Selected Areas in Communications, vol. 19, no. 6, pp. 10521060, 2001.

[25] J. H. Park, K.-Y. Han, and D.-H. Cho, "Reducing inter-cell handover events based on cell ID information in multi-hop relay systems," in Proceedings of the 65th IEEE Vehicular Technology Conference (VTC '07), pp. 743-747, Dublin, Ireland, 2007.

[26] A. J. Viterbi, A. M. Viterbi, K. S. Gilhousen, and E. Zehavi, "Soft handoff extends CDMA cell coverage and increases reverse link capacity," IEEE Journal on Selected Areas in Communications, vol. 12, no. 8, pp. 1281-1288, 1994.

[27] J.-H. Tsai and H.-D. Shih, "DC-20-GHz compact SPQT switch for butler matrix switched beam smart antenna system," Microwave and Optical Technology Letters, vol. 54, no. 9, pp. 2023-2026, 2012.

[28] L.-R. Hu and S. R. Stephen, "Personal communication systems using multiple hierarchical cellular overlays," IEEE Journal on Selected Areas in Communications, vol. 13, no. 2, pp. 406-415, 1995.

[29] S.-H. Wie, J. Jang, B. Shin, and D. Cho, "Handoff analysis of the hierarchical cellular system," IEEE Transactions on Vehicular Technology, vol. 49, no. 5, pp. 2027-2036, 2000.

[30] M. A. Marsan, G. Ginella, R. Maglione, and M. Meo, "Performance analysis of hierarchical cellular networks with generally distributed call holding times and dwell times," IEEE Transactions on Wireless Communications, vol. 3, no. 1, pp. 248-257, 2004.

[31] P. J. Smith, M. Shafi, and A. Tokeley, "On the effectiveness of channel segregation as a channel allocation method in a variety of cellular structures," IEEE Transactions on Vehicular Technology, vol. 49, no. 6, pp. 2234-2243, 2000.

[32] K. Okada and F. Kubota, "On dynamic channel assignment in cellular mobile radio systems," in Proceedings of the IEEE International Symposium on Circuits and Systems, pp. 938-941, June 1991.

[33] J. Kalvenes, J. Kennington, and E. Olinick, "Hierarchical cellular network design with channel allocation," European Journal of Operational Research, vol. 160, no. 1, pp. 3-18, 2005. 
[34] M. Suraya and A. M. Prasad, "An overview of smart antennas and its techniques beam forming and diversity," International Journal of Computational Engineering, vol. 2, no. 3, pp. 732-736, 2012.

[35] S. Saunders and A. Aragón-Zavala, Antennas and Propagation for Wireless Communication Systems, Wiley, 2007.

[36] A. Ahmad, "A CDMA network architecture using optimized sectoring," IEEE Transactions on Vehicular Technology, vol. 51, no. 3, pp. 404-410, 2002.

[37] K. Shalinee and L. Greenstein, "Capacity tradeoffs between macro-cell and micro-cell in a CDMA system: exact and approximate analyses," IEEE Transactions on Wireless Communications, vol. 2, no. 2, pp. 364-374, 2003. 

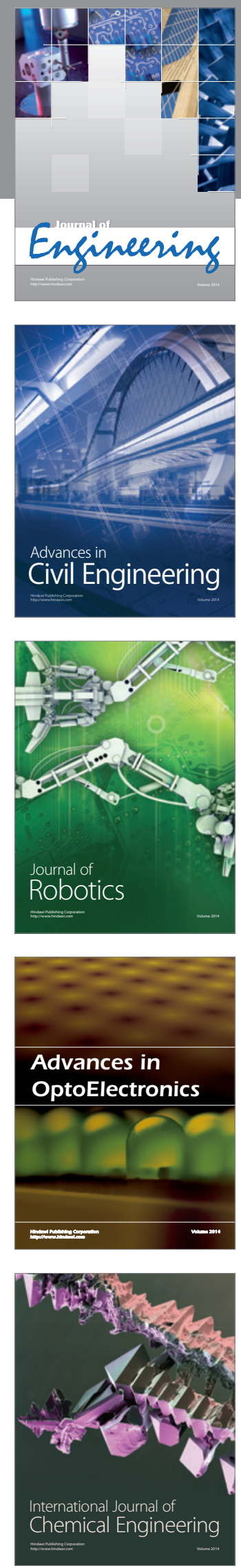

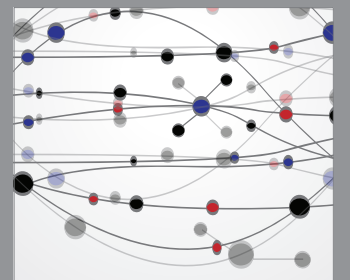

The Scientific World Journal
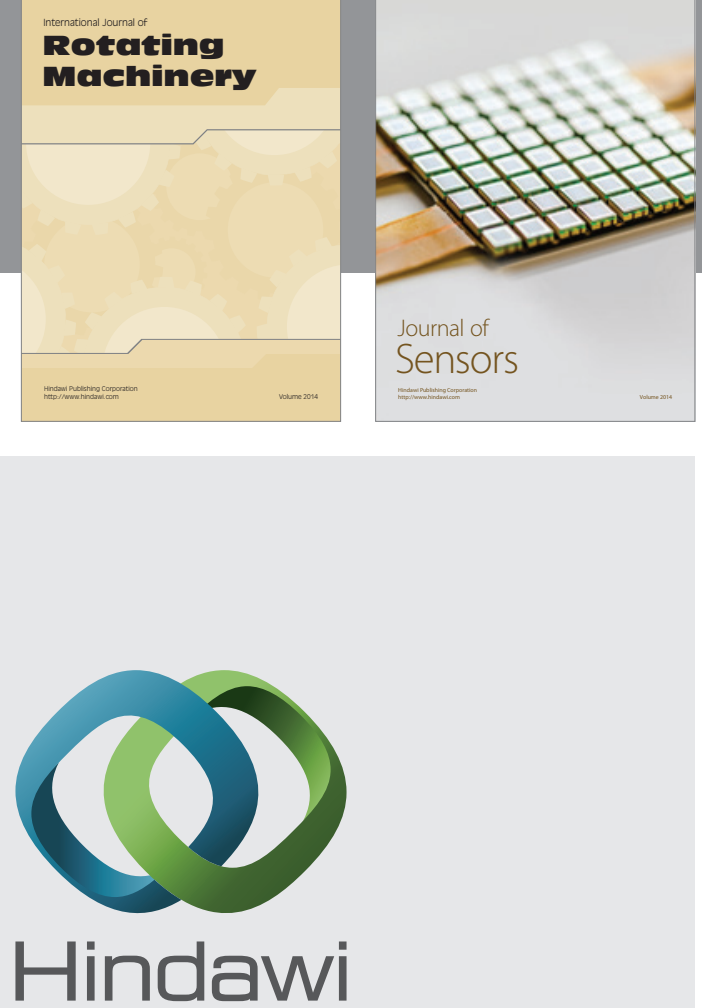

Submit your manuscripts at http://www.hindawi.com
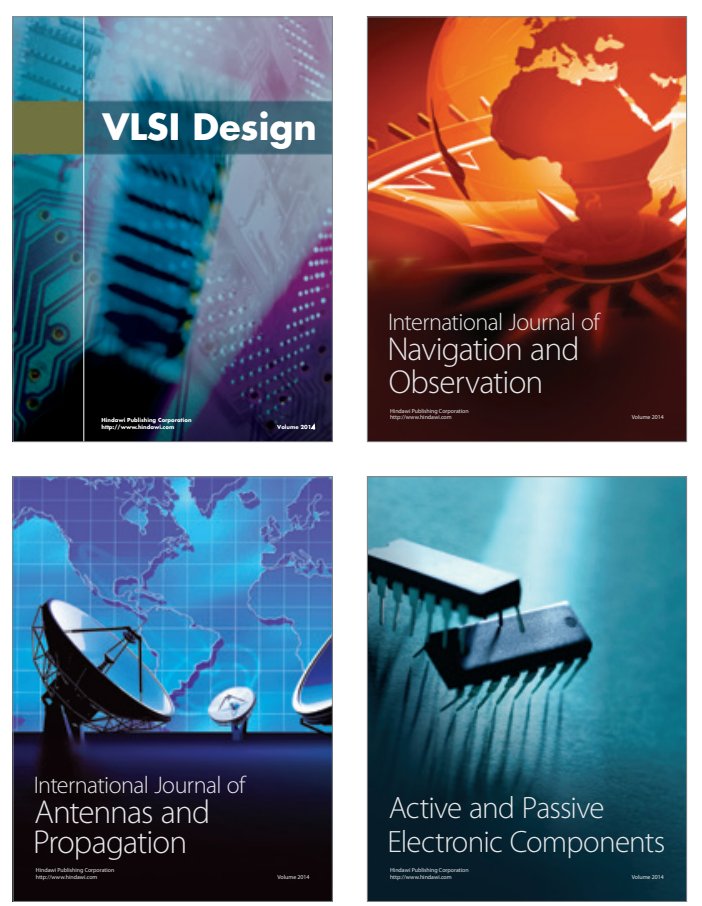
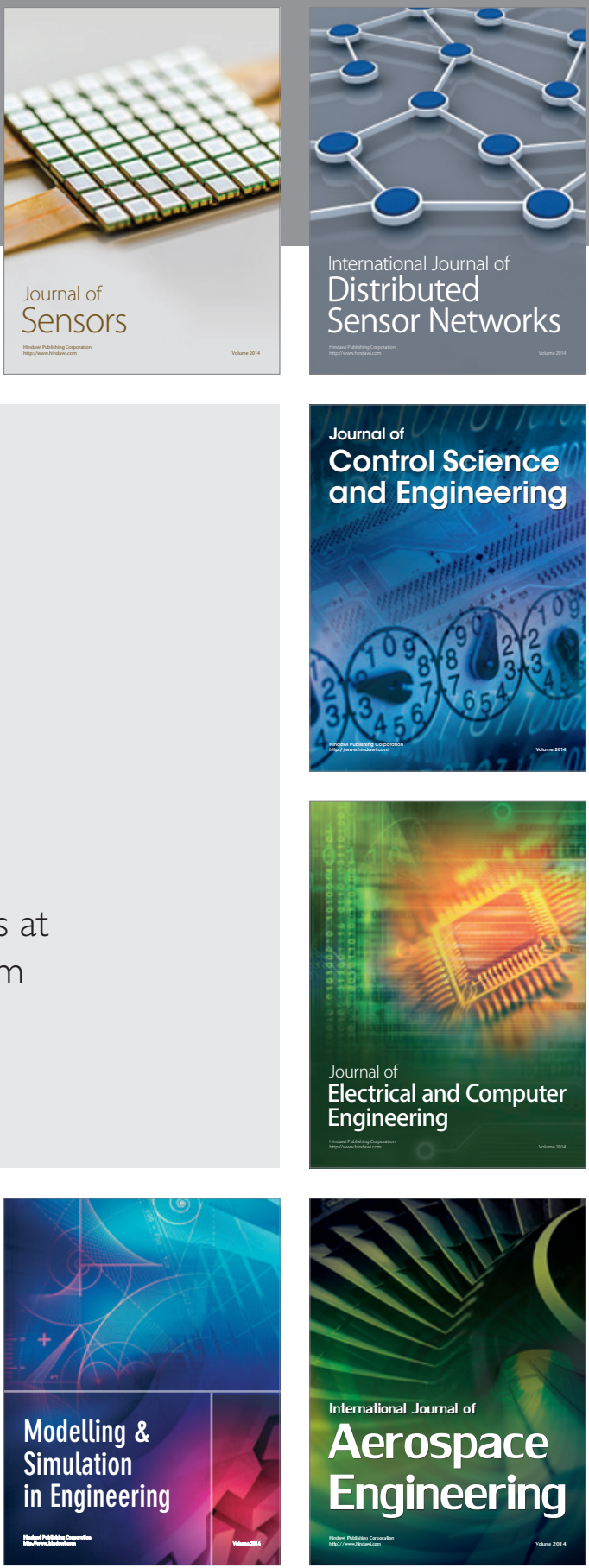

Journal of

Control Science

and Engineering
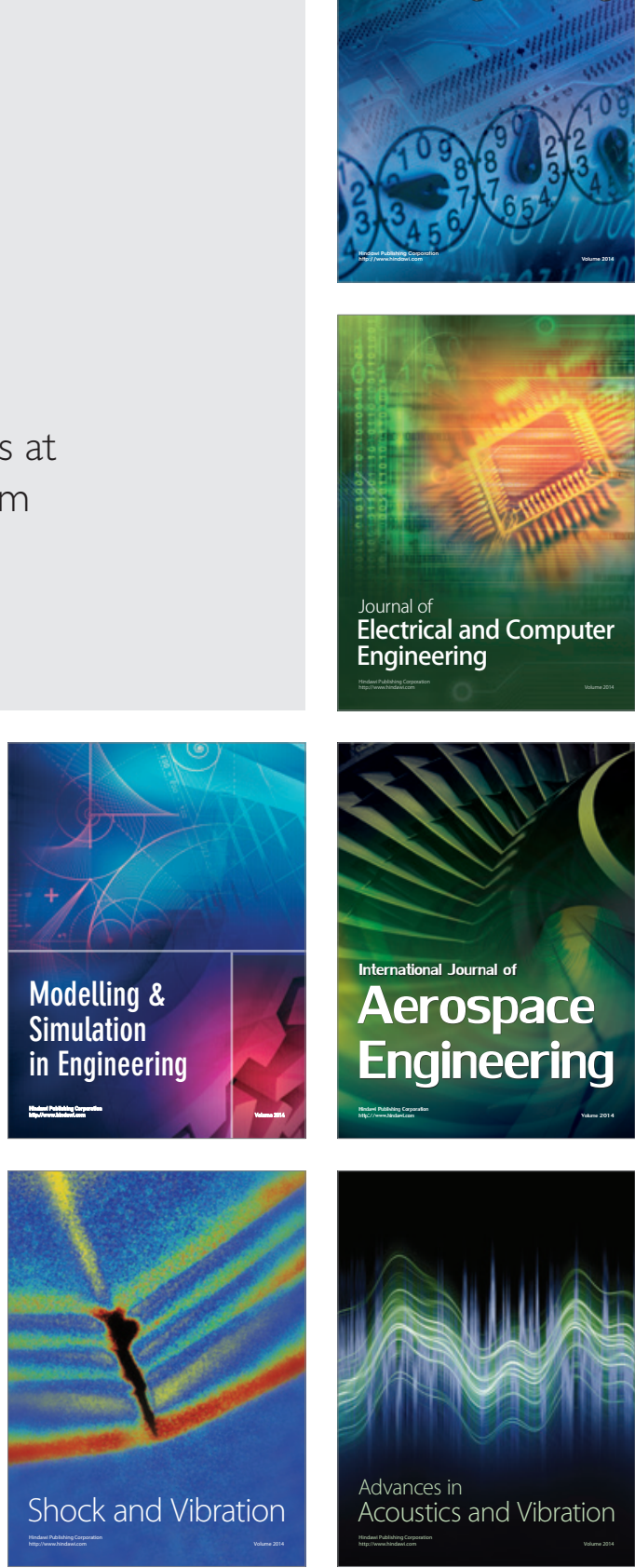